\title{
Comparações entre Meninos e Meninas no Zulliger - Sistema Compreensivo
}

\author{
Anna Elisa Villemor Amaral ${ }^{1}$ \\ Ana Carolina Zuanazzi $i^{1}$ \\ Fabiano Koich Miguel ${ }^{2}$ \\ André Pereira Gonçalves ${ }^{1}$ \\ ${ }^{1}$ Universidade São Francisco, Campinas, São Paulo, Brasil \\ ${ }^{2}$ Universidade Estadual de Londrina (UEL) Londrina, Paraná, Brasil
}

\begin{abstract}
Resumo
O objetivo deste estudo foi verificar se existe diferença no desempenho entre meninos e meninas no teste de Zulliger, pelo Sistema Compreensivo ZSC. A amostra constituiu-se de 623 crianças com idades entre 6 a 14 anos, provenientes da região Sudeste do Brasil, divididas em quatro faixas etárias, a saber, seis a sete anos, oito a nove, dez a onze e doze a quatorze anos. As médias foram comparadas por meio do teste $t$. Os resultados indicaram que algumas diferenças foram significativas, embora $o$ número de variáveis tenha sido reduzido consideravelmente, quando comparado com a literatura. Os achados são discutidos junto a estudos com técnicas projetivas e outros de personalidade. Conclui-se que, embora muitas variáveis sejam corroboradas na literatura, são necessários mais estudos com amostras mais homogêneas, por exemplo, controlando nível cognitivo e variáveis sociodemográficas.

Palavras-chave: medidas projetivas da personalidade, avaliação psicológica, personalidade, desenvolvimento
\end{abstract}

\section{Comparisons Between Boys and Girls in Zulliger - Comprehensive System}

\begin{abstract}
The objective of this study was to verify possible differences in performance between boys and girls in the Zulliger Comprehensive System (ZSC). The sample consisted of 623 children aged from 6 to 14, from the Southeast region of Brazil, divided into four age groups: six to seven years, eight to nine, ten to eleven, and twelve to fourteen years. The means were compared using the $t$-test. The results indicated that some differences remained significant even after the Bonferroni correction, although the number of variables was reduced considerably when compared to the literature. The findings are discussed together with studies with projective techniques as well as other personality techniques. It was concluded that, although many variables were corroborated in the literature, more studies with more homogenous samples are needed, including, for example, control for the cognitive level and sociodemographic variables.

Keywords: projective personality measures; psychological assessment; personality; development.
\end{abstract}

Las Comparaciones Entre Niños y Niñas en Zulliger - Sistema Comprehensivo

\section{Resumen}

El objetivo de este estudio fue verificar si existe diferencia en el rendimiento entre niños y niñas en la prueba de Zulliger, por el Sistema Comprensivo ZSC. Las medias se compararon usando el $t$ test. La muestra estuvo conformada por 623 niños con edades entre 6 y 14 años, provenientes de la región Sudeste de Brasil, dichos niños fueron divididos en cuatro grupos de edad: de seis a siete, de ocho a nueve, de diez a once y de doce a catorce años. Los resultados indicaron que, incluso con la corrección de Bonferroni, algunas diferencias se mantuvieron significativas, aunque el número de variables se redujo considerablemente en comparación con la literatura. Los hallazgos son discutidos junto con estudios con técnicas proyectivas y otras técnicas de personalidad. Se concluye que, aunque muchas variables se corroboran en la literatura, son necesarios más estudios con muestras más homogéneas, por ejemplo, controlando el nivel cognitivo y las variables sociodemográficas.

Palabras clave: medidas de personalidad proyectiva; evaluación psicológica; personalidad; desarrollo.

\section{Introdução}

Estudos sobre personalidade com crianças e adolescentes têm sido conduzidos com o objetivo de identificar traços característicos dessa população, bem como mudanças ao longo do desenvolvimento psicossocial (Caspi, Roberts, \& Shiner, 2005; Denham, Wyatt, Bassett, Echeverria, \& Knox, 2009; Klimstra, Hale III, Raaijmakers, Branje, \& Meeus, 2009; Soto, 2015). Por meio de tal caracterização, seria possível avaliar mais coerentemente aspectos do desenvolvimento infantil e assim identificar precocemente potencialidades e dificuldades da criança ou adolescente, tendo em vista que fatores da personalidade estariam fortemente relacionados a comportamentos delinquentes (Heaven, 1996), abuso de drogas (Ruiz, Pincus, \& Dicknson, 2003), entre outros. Supõe-se que seria possível propor intervenções mais apropriadas, minimizando problemas 
psicopatológicos e maximizando características positivas para um crescimento saudável (Branje, Van Lieshout, \& Gerris, 2007; Meyer, Giromini, Viglione, Reese, \& Mihura, 2014).

Pesquisas sobre personalidade incluem a investigação de diferenças entre meninos e meninas (Branje, Van Lieshout, \& Gerris, 2007; Klimstra et al., 2009; McCrae et al., 2002). As diferenças entre sexo ocorreriam em períodos específicos do desenvolvimento humano, sendo estes presentes mais acentuadamente na infância e adolescência, reduzindo drasticamente na fase adulta (Branje et al., 2007; Meyer et al., 2014). Isso se deve tanto ao desenvolvimento psicossocial quanto às alterações biológicas, como maturação neurológica e hormonal.

Os achados sobre personalidade na infância e adolescência, porém, não têm sido coerentes, havendo acentuadas diferenças entre amostras. Se por um lado Branje, Van Lieshout e Gerris (2007), usando um instrumento de autorrelato (Big Five Personality Dimensions), encontraram evidências de que os meninos seriam mais extrovertidos do que meninas, McCrae et al. (2002) verificaram resultados opostos para os mesmos períodos do desenvolvimento, observando, adicionalmente, que meninas apresentaram maior nível de amabilidade e menor estabilidade emocional quando comparado aos meninos. Já Klimstra, Hale III, Raaijmakers, Branje e Meeus (2009) verificaram que meninas apresentavam maiores níveis de estabilidade emocional. Ainda não é claro se essas inconsistências na literatura seriam decorrentes das amostras, de grupos etários específicos, diferenças socioculturais, dos instrumentos utilizados ou mesmo de uma combinação desses fatores (Klimstra et al., 2009; Soto, John, Gosling, \& Potter, 2011). Assim, são necessárias mais investigações com intuito de analisar se realmente existe diferença entre meninos e meninas a fim de possibilitar uma compreensão mais aprofundada e com melhores estratégias de intervenção nessa faixa etária.

No que tange aos instrumentos utilizados para essas investigações, nota-se que a maioria dos estudos com personalidade em crianças e adolescentes utiliza medidas de auto ou heterorrelato, e um menor número de estudos foram conduzidos com técnicas projetivas (Tavella \& Villemor-Amaral, 2014). Os instrumentos projetivos facilitariam a investigação de determinadas características, como a personalidade, por se tratarem de estímulos pouco estruturados, diminuindo o controle, manipulação de respostas e efeitos de uma percepção pouco realista de si (Chabert, 2004; Di Domenico Grazziotin \& Scortegagna, 2016).

Dentre os instrumentos projetivos com estudos de normatização para crianças e disponíveis para uso no contexto brasileiro, encontra-se o teste das manchas de tinta de Rorschach. Estudos com esse teste apresentaram resultados condizentes com o esperado para o desenvolvimento psicossocial, emocional/ afetivo e processamento cognitivo de crianças e adolescente de 5 a 14 anos, sendo favorável seu uso nessa população (Cury-Jacquemin, 2012; Fernandes, 2010; Nascimento, Pedroso, \& Souza, 2009; Raspantini, 2010; Resende, Carvalho, \& Martins, 2012; Ribeiro, Semer, \& Yazigi, 2011).

Outro teste, baseado nos princípios do Rorschach, que se encontra em desenvolvimento de normas para crianças e adolescentes, é o Teste de Zulliger. Trata-se de uma técnica projetiva bastante utilizada no Brasil, em diversos contextos (Angelini \& Oliveira, 2003; Cardoso, Gomes, Pacheco, \& Dias-Viana, 2018; Di Domenico Grazziotin \& Scortegagna, 2016; Ferreira \& Villemor-Amaral, 2005; Franco \& Villemor-Amaral, 2012; Gregoleti \& Scortegagna, 2017; Vaz, 2000; Villemor-Amaral \& Machado, 2011; Villemor-Amaral, Santos, \& Noronha, 2009). Atualmente, para uso com público infantil, está disponível em dois sistemas de aplicação, codificação e interpretação, o Sistema de Classificação Klopfer (Vaz, 2004) e o Sistema Compreensivo (ZSC; Villemor-Amaral \& Primi, 2009).

O Zulliger, de acordo com o Sistema Compreensivo, que será utilizado neste estudo, fornece dados sobre seis áreas do funcionamento que são o Processamento da Informação, Mediação, Ideação, Processamento Emocional/Afetivo, Percepção Interpessoal e Autoimagem e a área Controle e Tolerância ao Estresse. As três primeiras, refletem aspectos do funcionamento cognitivo e são denominadas tríade cognitiva. A primeira área, Processamento da Informação, refere-se ao modo como a pessoa apreende os estímulos ao seu redor. Reúne variáveis sobre o que é favorecido ao receber uma informação nova. Contido nesse grupo, estão indicadores sobre o modo de apreensão da informação, abertura às experiências e recursos intelectuais (Villemor-Amaral \& Primi, 2009).

O segundo grupo, Mediação, está relacionado ao processo de tradução da informação apreendida. As variáveis reunidas nesse conjunto são interpretadas de acordo com o quão convencional ou idiossincrático é a representação que o sujeito tem da realidade. A área Ideação, por sua vez, está ligada à interpretação que o 
indivíduo tem sobre as experiências de vida. Indicam o quão lógica, coerente e flexível é tal percepção (Villemor-Amaral \& Primi, 2009).

A área Processamento Emocional/Afetivo associa-se à forma como as emoções interferem no julgamento e relacionamento com o outro. Esse grupo é apresentado em subcategorias que são a capacidade de modular os afetos, a constrição emocional, indicadores de tendência à autonomia ou à negação, intelectualização, entre outros. A área Percepção Interpessoal e Autoimagem é interpretada como a percepção do indivíduo sobre as relações interpessoais e sobre si, respectivamente, podendo ser essa apreensão mais integrativa ou mais incompleta/fantasiosa. Por fim, o Controle e Tolerância ao Estresse informam sobre o estilo desenvolvido pelo sujeito para lidar com situações diversas, favorecendo ações mais previsíveis no manejo de circunstâncias estressoras, resolução de problemas e tomada de decisões (Villemor-Amaral \& Primi, 2009).

Com o objetivo de localizar pesquisas sobre o uso do Zulliger na população infantil e adolescente, os autores do presente estudo realizaram uma busca nas bases de dados da BVS Psicologia com o descritor "Zulliger". Após a exclusão de artigos duplicados, de livros e outros materiais não revisados por pares, foram identificados apenas nove estudos que utilizam o Zulliger em amostras infantis. Além disso, também foi localizada uma dissertação que propôs um estudo de normatização para faixa etária dos 7 aos 14 anos (Carvalho, 2015). Um deles foi baseado no Sistema Klopfer (Rodrigues \& Alchieri, 2009) e os demais, no Sistema Compreensivo (Biasi \& Villemor-Amaral, 2016; Cardoso, Gomes, \& Vieira, 2018; Carvalho \& Resende, 2018; Rodrigues \& Alchieri, 2009; Tavella \& Villemor-Amaral, 2014; Villemor-Amaral, Pavan, Tavella, Cardoso, \& Biasi, 2016; Villemor-Amaral \& Quirino, 2013; Villemor-Amaral \& Vieira, 2016).

Os estudos apontaram para acurácia do teste em diferenciar grupos de acordo com características específicas, como criatividade (Tavella \& Villemor-Amaral, 2014) e traços como sociabilidade (Biasi \& Villemor-Amaral, 2016). Aliado a isso, o Zulliger mostrou-se adequado para diferenciar grupos etários de acordo com a maturidade relacional, emocional e intelectual (Cardoso, Gomes, \& Vieira, 2018; Carvalho, 2015; Villemor-Amaral et al., 2016; Villemor-Amaral \& Vieira, 2016), indicando as evidências de validade de critério (American Educational Research Association, American Psychological Association, \& National Council on Measurement in Education, 2014).
Tais pesquisas iniciais com a população infantil e adolescente têm mostrado que o teste de Zulliger seria uma ferramenta adequada para distinguir diferenças entre grupos, seja em características de personalidade, como também relacionais e intelectuais. Hipotetiza-se que esse instrumento também seria capaz de verificar a existência de diferenças entre meninos e meninas, aspectos cujos resultados ainda são pouco coerentes na literatura. Para verificar essa hipótese, este estudo exploratório foi delineado com objetivo de verificar se existe diferença no desempenho de meninos e meninas no teste de Zulliger, pelo Sistema Compreensivo ZSC a fim de contribuir para melhor uso dos resultados em intervenções clínicas.

\section{Método}

\section{Participantes}

Participaram desse estudo 623 crianças, ambos os sexos, em sua maioria meninas $(54,6 \%)$ com idades de 6 a 14 anos e estudantes de escolas públicas (79,6\%) e privadas $(20,4 \%)$ da Região Sudeste do Brasil. Os participantes foram agrupados em quatro faixas etárias, dos 6 aos 7 anos ( $n=127$, sendo 59,8\% meninas), dos 8 aos 9 anos ( $n=141$, sendo 58,9\% meninas), dos 10 aos 11 anos ( $n=215$, sendo 53,5\% meninas), dos 12 aos 14 anos $(n=140$, sendo $47,1 \%$ meninas $)$.

\section{Instrumentos}

\section{Questionário Sociodemográfico}

Compuseram o questionário sociodemográfico informações referentes à data de nascimento, ao ano escolar, ao nome da escola e ao tipo de escola que o respondente estava matriculado (pública ou privada), além de campo para inclusão do código do participante e sexo. O levantamento desses dados foi feito com objetivo de caracterizar a amostra.

\section{Teste de Zulliger no Sistema Compreensivo}

Esse instrumento é composto por uma sequência fixa de três pranchas com borrões de tinta, para os quais se solicita que o sujeito diga com o que se parecem. Após a apresentação de todas as pranchas, uma após a outra, cada resposta dada é revista perguntando-se ao participante onde viu e quais aspectos da mancha foram relevantes para tal percepção. Não há limite de respostas para cada cartão (Villemor-Amaral \& Primi, 2009).

Os códigos atribuídos são submetidos a cálculos, resultando em indicadores diversos agrupados 
em seis áreas do funcionamento psicológico que são o Processamento da Informação, Mediação, Ideação, Processamento Emocional/Afetivo, Percepção Interpessoal e Autoimagem e Controle e Tolerância ao Estresse. O teste foi estudado com crianças por Tavella e Villemor-Amaral (2014), Villemor-Amaral et al. (2016) Villemor-Amaral e Quirino (2013) Villemor-Amaral e Vieira (2016) que verificaram evidências de validade de critério para uso com essa população, sendo que a precisão variou entre 0,88 e 1,00 (coeficiente Kappa).

\section{Procedimentos}

Foi solicitado a algumas escolas públicas e privadas do Sudeste do Brasil a autorização para a coleta de dados dentro das instituições. Após autorização das instituições, o projeto foi submetido e aprovado pelo Comitê de Ética em Pesquisas com Seres Humanos (CAAE: 0078.0.142.000-07). Foram enviados aos pais dos alunos o Termo de Consentimento Livre e Esclarecido para que estes pudessem estar cientes da pesquisa e autorizassem a participação das crianças. As crianças cujos pais responderam autorizando participação foram convidadas a participar da pesquisa e, após seu assentimento, responderam em uma sessão individual e privativa ao teste de Zulliger. As aplicações tiveram duração aproximada de 30 minutos.

\section{Análise de Dados}

Foi feita a comparação do valor médio de variáveis do Zulliger por sexo de acordo com a faixa etária por meio do teste $t$ de Student de medidas independentes. Para criação dos grupos etários, foi tomado como base o ano escolar das crianças, considerando esta ser uma variável importante no processo de desenvolvimento cognitivo e também social. Após essa análise, foi feita correção de Bonferroni para cada faixa etária considerando o valor base $p \leq 0,05$. O uso dessa ferramenta é indicado quando se faz múltiplas comparações numa mesma amostra, uma vez que a multiplicidade de análises geraria maior probabilidade de erro tipo I, ou seja, quando a hipótese nula é verdadeira, mas é rejeitada, ocorrendo em falsos positivos. Considerando a necessidade de redução de erro tipo I nesse tipo de análise, adotou-se esse procedimento de correção também nos estudos que foram usados para comparação e discussão com os achados da presente pesquisa. Assim, sempre que os estudos referenciados tenham apresentado o valor de $p$ e o tamanho do grupo de comparação, foi feita correção de Bonferroni. Assim, espera-se uma melhor comparação entre os achados. Adicionalmente, foi calculado o tamanho do efeito utilizando o coeficiente $d$ de Cohen, tendo como valores de referência por volta de 0,20 (pequeno), ao redor de 0,50 (moderado) e próximo a 0,80 (grande) (Cohen, 1992).

\section{Resultados}

As tabelas 1, 2, 3 e 4 apresentam os valores médios, desvios padrão, valor de $t$, nível de significância $(p)$ e tamanho do efeito (d) das variáveis cujas diferenças foram estatisticamente significativas para cada faixa etária, comparando-se meninos e meninas. Estão assinaladas com asterisco $\left(^{*}\right)$ as variáveis consideradas para análise após a correção de Bonferroni.

Embora seis variáveis tenham apresentado valor $p$ $\leq 0,05$, sendo uma relacionada ao conjunto Processamento da Informação (DQ+) e as demais relacionadas à Percepção Interpessoal e Autoimagem $[\mathrm{H},(\mathrm{H}), \mathrm{Hd}$, Hh, PHR], pela correção de Bonferroni foram considerados significativos apenas valores $p \leq 0,008$. Dessa forma, observou-se que nenhuma variável apresentou diferença significativa após ajuste de Bonferroni, não havendo diferenças no desempenho entre meninos e meninas nessa faixa etária. Porém, observando-se o tamanho de efeito das comparações, cinco variáveis apresentaram tamanho pequeno enquanto a variável DQ+ apresentou tamanho moderado.

$\mathrm{Na}$ faixa etária dos oito aos nove anos, há nove variáveis com valor de $p \leq 0,05$, porém pelo ajuste de Bonferroni foram considerados significativos apenas valores $p \leq 0,005$, indicando apenas três variáveis que atendem a essa especificação. Uma das variáveis, Bl (conteúdo de sangue), faz parte do conjunto Percepção Interpessoal e Autoimagem e foi mais produzida pelos meninos. O tamanho de efeito dessa variável foi moderado. As duas outras variáveis, XA\% e WDA\%, referem-se ao conjunto Mediação e seus índices foram maiores nas meninas, com tamanhos de efeito moderado e forte, respectivamente.

Doze variáveis apresentaram valor de $p \leq 0,05$ na faixa etária dos 10 aos 11 anos. Pela correção de Bonferroni, foram considerados significativos apenas valores $p \leq 0,004$. Dessa forma, apenas as variáveis ma e m, relacionadas ao conjunto Processamento Emocional/Afetivo, apresentaram diferenças significativas sendo mais produzidas pelos meninos. Os tamanhos de efeito dessas variáveis foram pequenos e moderados, respectivamente.

Por fim, na faixa etária entre 12 e 14 anos, cinco variáveis apresentaram valor de $p \leq 0,05$, porém, pelo 
Tabela 1.

Comparação do Valor Médio em Crianças de Faixa Etária de 6 a 7 Anos por Sexo

\begin{tabular}{|c|c|c|c|c|c|c|c|}
\hline & Variável & Grupo & Média & $D P$ & $t$ & $p$ & $d$ \\
\hline \multirow{2}{*}{$\begin{array}{l}\text { Processamento da } \\
\text { Informação }\end{array}$} & $\mathrm{DQ}^{+}$ & Feminino & 0,87 & 1,10 & $-2,015$ & 0,046 & 0,49 \\
\hline & & Masculino & 1,25 & 1,00 & & & \\
\hline \multirow{10}{*}{$\begin{array}{l}\text { Percepção } \\
\text { Interpessoal e } \\
\text { Autoimagem }\end{array}$} & $\mathrm{H}$ & Feminino & 0,86 & 0,78 & 2,148 & 0,034 & 0,40 \\
\hline & & Masculino & 0,57 & 0,67 & & & \\
\hline & $(\mathrm{H})$ & Feminino & 0,42 & 0,73 & $-2,376$ & 0,019 & 0,41 \\
\hline & & Masculino & 0,78 & 0,99 & & & \\
\hline & $\mathrm{Hd}$ & Feminino & 0,22 & 0,51 & $-2,077$ & 0,040 & 0,36 \\
\hline & & Masculino & 0,47 & 0,83 & & & \\
\hline & $\mathrm{Hh}$ & Feminino & 0,04 & 0,20 & $-2,224$ & 0,028 & 0,37 \\
\hline & & Masculino & 0,20 & 0,57 & & & \\
\hline & PHR & Feminino & 0,61 & 0,88 & $-2,467$ & 0,015 & 0,43 \\
\hline & & Masculino & 1,04 & 1,10 & & & \\
\hline
\end{tabular}

Nota. $D P=$ Desvio Padrão, $t=$ valor resultante do teste $t$ de Student, $p=$ nível de significância, $d=\mathrm{d}$ de Cohen para mensuração do tamanho do efeito.

Tabela 2.

Comparação do Valor Médio em Crianças de Faixa Etária de 8 a 9 Anos por Sexo

\begin{tabular}{|c|c|c|c|c|c|c|c|}
\hline & Variável & Grupo & Média & $D P$ & $t$ & $p$ & $d$ \\
\hline \multirow{4}{*}{$\begin{array}{l}\text { Processamento } \\
\text { Emocional/Afetivo }\end{array}$} & $\mathrm{YF}$ & Feminino & 0,02 & 0,15 & $-2,019$ & 0,045 & 0,32 \\
\hline & & Masculino & 0,10 & 0,31 & & & \\
\hline & $\mathrm{Bl}$ & Feminino & 0,00 & 0,00 & $-3,351$ & $0,001^{*}$ & 0,47 \\
\hline & & Masculino & 0,12 & 0,33 & & & \\
\hline \multirow{4}{*}{$\begin{array}{l}\text { Percepção Interpessoal e } \\
\text { Autoimagem }\end{array}$} & FQo com & Feminino & 0,34 & 0,63 & $-2,202$ & 0,029 & 0,37 \\
\hline & M & Masculino & 0,59 & 0,70 & & & \\
\hline & $(\mathrm{H})$ & Feminino & 0,33 & 0,56 & $-2,552$ & 0,012 & 0,41 \\
\hline & & Masculino & 0,66 & 0,96 & & & \\
\hline \multirow[t]{2}{*}{ Ideação } & WSum6 & Feminino & 1,60 & 2,38 & $-2,742$ & 0,007 & 0,44 \\
\hline & & Masculino & 3,07 & 3,96 & & & \\
\hline \multirow[t]{6}{*}{ Mediação } & $\mathrm{XA} \%$ & Feminino & 0,79 & 0,17 & 2,882 & $0,005^{*}$ & 0,47 \\
\hline & & Masculino & 0,70 & 0,21 & & & \\
\hline & WDA\% & Feminino & 0,81 & 0,18 & 3,259 & $0,001^{*}$ & 0,53 \\
\hline & & Masculino & 0,70 & 0,23 & & & \\
\hline & $\mathrm{X}-\%$ & Feminino & 0,21 & 0,17 & $-2,644$ & 0,009 & 0,47 \\
\hline & & Masculino & 0,30 & 0,21 & & & \\
\hline \multirow{2}{*}{$\begin{array}{l}\text { Processamento da } \\
\text { Informação }\end{array}$} & FQ- em & Feminino & 1,18 & 1,17 & $-2,363$ & 0,020 & 0,10 \\
\hline & WD & Masculino & 1,06 & 1,18 & & & \\
\hline
\end{tabular}

Nota. $D P=$ Desvio Padrão, $t=$ valor resultante do teste $t$ de Student, $p=$ nível de significância, $d=\mathrm{d}$ de Cohen para mensuração do tamanho do efeito. 
Tabela 3.

Comparação do Valor Médio em Crianças de Faixa Etária de 10 a 11 Anos por Sexo

\begin{tabular}{|c|c|c|c|c|c|c|c|}
\hline & Variável & Grupo & Média & $D P$ & $t$ & $p$ & $d$ \\
\hline \multirow{10}{*}{$\begin{array}{l}\text { Processamento } \\
\text { emocional/afetivo }\end{array}$} & \multirow[t]{2}{*}{$\mathrm{mp}$} & Feminino & 0,17 & 0,42 & \multirow[t]{2}{*}{2,224} & \multirow[t]{2}{*}{0,027} & \multirow[t]{2}{*}{0,32} \\
\hline & & Masculino & 0,06 & 0,24 & & & \\
\hline & \multirow[t]{2}{*}{$\mathrm{ma}$} & Feminino & 0,01 & 0,93 & \multirow[t]{2}{*}{$-3,083$} & \multirow[t]{2}{*}{$0,002^{*}$} & \multirow[t]{2}{*}{0,13} \\
\hline & & Masculino & 0,10 & 0,30 & & & \\
\hline & \multirow[t]{2}{*}{$\mathrm{Mp}$} & Feminino & 0,06 & 0,27 & \multirow[t]{2}{*}{$-1,970$} & \multirow[t]{2}{*}{0,050} & \multirow[t]{2}{*}{0,26} \\
\hline & & Masculino & 0,15 & 0,39 & & & \\
\hline & \multirow[t]{2}{*}{$\mathrm{m}$} & Feminino & 0,07 & 0,32 & \multirow[t]{2}{*}{$-3,116$} & \multirow[t]{2}{*}{$0,002^{*}$} & \multirow[t]{2}{*}{0,41} \\
\hline & & Masculino & 0,25 & 0,52 & & & \\
\hline & \multirow[t]{2}{*}{ FC } & Feminino & 0,15 & 0,30 & \multirow[t]{2}{*}{$-2,166$} & \multirow[t]{2}{*}{0,031} & \multirow[t]{2}{*}{0,30} \\
\hline & & Masculino & 0,29 & 0,57 & & & \\
\hline \multirow{8}{*}{$\begin{array}{l}\text { Percepção interpessoal e } \\
\text { autoimagem }\end{array}$} & \multirow[t]{2}{*}{ A } & Feminino & 3,57 & 1,80 & \multirow[t]{2}{*}{2,305} & \multirow[t]{2}{*}{0,022} & \multirow[t]{2}{*}{0,24} \\
\hline & & Masculino & 3,01 & 1,72 & & & \\
\hline & \multirow[t]{2}{*}{$\mathrm{Ad}$} & Feminino & 0,18 & 0,45 & \multirow[t]{2}{*}{$-2,176$} & \multirow[t]{2}{*}{0,031} & \multirow[t]{2}{*}{0,29} \\
\hline & & Masculino & 0,37 & 0,79 & & & \\
\hline & \multirow[t]{2}{*}{$\mathrm{Na}$} & Feminino & 0,14 & 0,35 & \multirow[t]{2}{*}{$-2,410$} & \multirow[t]{2}{*}{0,017} & 0,32 \\
\hline & & Masculino & 0,30 & 0,61 & & & \\
\hline & Isolamento & Feminino & 0,15 & 0,15 & $-1,974$ & 0,050 & 0,26 \\
\hline & & Masculino & 0,20 & 0,22 & & & \\
\hline Mediação & P & Feminino & 1,48 & 1,00 & 2,065 & 0,040 & 0,28 \\
\hline & & Masculino & 1,19 & 1,04 & & & \\
\hline Processamento da & W\% & Feminino & 18,69 & 19,67 & $-2,516$ & 0,013 & 0,34 \\
\hline Informação & & Masculino & 26,30 & 24,63 & & & \\
\hline & $\mathrm{D} \%$ & Feminino & 60,83 & 20,82 & 2,468 & 0,014 & 0,33 \\
\hline & & Masculino & 53,39 & 23,33 & & & \\
\hline
\end{tabular}

Nota. $D P=$ Desvio Padrão, $t=$ valor resultante do teste $t$ de Student, $p=$ nível de significância $d=\mathrm{d}$ de Cohen para mensuração do tamanho do efeito.

ajuste de Bonferroni, foram considerados significativos apenas valores $p \leq 0,01$. Dessa forma, apenas a variável $\mathrm{Xu} \%$, da área Mediação, foi considerada significativamente diferente e com tamanho de efeito forte, encontrando-se em maior nível nas meninas.

\section{Discussão}

O objetivo deste estudo foi verificar se existe diferença no desempenho entre meninos e meninas no teste de Zulliger, pelo Sistema Compreensivo ZSC, agrupados em quatro faixas etárias. Dessa forma, espera-se contribuir para melhor compreensão de peculiaridades nessa faixa etária quando se trata da diferença entre meninos e meninas e, com isso, contribuir para propostas de intervenções clínicas mais condizentes. Diferentemente dos demais estudos que fizeram comparações múltiplas com o Zulliger em amostras infantis, este utilizou métodos de análise mais rigorosos com a finalidade de reduzir erros de tipo I. Com isso, verificou-se que, ao utilizar o método de correção de Bonferroni, poucas variáveis puderam ser consideradas com diferenças estatisticamente significativas entre os grupos. Também foram considerados os tamanhos de efeito das comparações.

Os dados encontrados na presente pesquisa são parcialmente relacionados aos de Carvalho (2015). A pesquisadora encontrou diferenças significativas entre 
Tabela 4.

Comparação do Valor Médio em Crianças de Faixa Etária de 12 a 14 Anos por Sexo

\begin{tabular}{|c|c|c|c|c|c|c|c|}
\hline \multirow{11}{*}{ Mediação } & Variável & Grupo & Média & $D P$ & $t$ & $p$ & $d$ \\
\hline & \multirow[t]{2}{*}{ WD- } & Feminino & 0,56 & 0,82 & \multirow[t]{2}{*}{$-2,423$} & \multirow[t]{2}{*}{0,017} & \multirow[t]{2}{*}{0,41} \\
\hline & & Masculino & 1,00 & 1,25 & & & \\
\hline & \multirow[t]{2}{*}{$\mathrm{XA} \%$} & Feminino & 0,84 & 0,16 & \multirow[t]{2}{*}{2,048} & \multirow[t]{2}{*}{0,042} & \multirow[t]{2}{*}{0,34} \\
\hline & & Masculino & 0,78 & 0,19 & & & \\
\hline & \multirow[t]{2}{*}{ WDA\% } & Feminino & 0,90 & 0,14 & \multirow[t]{2}{*}{2,420} & \multirow[t]{2}{*}{0,017} & \multirow[t]{2}{*}{0,41} \\
\hline & & Masculino & 0,83 & 0,19 & & & \\
\hline & \multirow[t]{2}{*}{$\mathrm{Xu} \%$} & Feminino & 0,38 & 0,19 & \multirow[t]{2}{*}{2,615} & \multirow[t]{2}{*}{$0,010^{*}$} & \multirow[t]{2}{*}{0,47} \\
\hline & & Masculino & 0,29 & 0,19 & & & \\
\hline & \multirow[t]{2}{*}{ S- } & Feminino & 0,60 & 0,24 & \multirow[t]{2}{*}{$-2,455$} & \multirow[t]{2}{*}{0,015} & \multirow[t]{2}{*}{0,92} \\
\hline & & Masculino & 0,23 & 0,51 & & & \\
\hline
\end{tabular}

Nota. $D P=$ Desvio Padrão, $t=$ valor resultante do teste $t$ de Student, $p=$ nível de significância, $d=\mathrm{d}$ de Cohen para mensuração do tamanho do efeito.

meninos e meninas na faixa dos sete aos nove anos em variáveis relacionadas a cognição (como DQv, FQnone) e relacionadas a Percepção Interpessoal e Autoimagem (como presença de Fd, conteúdo de comida). Porém, há dois pontos a serem observados. O primeiro é que a autora não utilizou o procedimento estatístico de ajuste de Bonferroni, resultando em mais relações significativas, porém não protegidas de erros tipo I. O segundo ponto a ser levantado relaciona-se ao de Processamento da Informação. No estudo de Carvalho (2015), as variáveis que apresentaram nível de significância são diferentes das aqui encontradas, mesmo quando não se considera a correção de Bonferroni. Nesse sentido, no presente estudo, os meninos apresentaram mais $\mathrm{DQ}+$ do que as meninas, indicando um processamento cognitivo mais elaborado e melhor desenvolvido na capacidade de integrar e processar diferentes elementos. Já na pesquisa de Carvalho (2015), foi encontrado algo na direção oposta, meninos com indicadores de menores recursos para processamento de informações (mais DQv e mais FQnone).

O estudo de Fernandes (2010) verificou que meninos de 6 a 8 anos apresentaram mais respostas dos tipo F- do que as meninas no Rorschach, sistema Escola Francesa. Essa variável é interpretada como uma apreensão da realidade pouco precisa e idiossincrática, o que iria ao encontro dos dados observados no estudo de Carvalho (2015). Porém, novamente, quando se aplica o ajustamento de Bonferroni na amostra de Fernandes (2010), essa variável deixa de ser considerada dentro dos limites de significância aceitável. Há duas hipóteses para tais divergências e convergências. Primeiramente, as faixas etárias consideradas neste estudo e nos de Carvalho (2015) e Fernandes (2010) são diferentes, sendo aqui considerada a faixa de seis a sete, no estudo de Carvalho (2015) dos 7 aos 9 anos e em Fernandes (2010) dos 6 aos 8 anos e são usados instrumentos de mancha de tintas distintos (Zulliger e Rorschach, respectivamente). Por outro lado, também seria possível supor a não existência de diferenças entre meninos e meninas referentes ao modo como a informação é processada, já que duas pesquisas muito similares encontraram resultados opostos. Isso poderia ocorrer, pois uma análise estatística menos rigorosa apontou diferenças que são opostas. Além disso, variáveis socioculturais poderiam estar mediando os resultados. Já quando se usa um método mais criterioso, essa diferença deixa de existir. Adicionalmente, em termos de desenvolvimento infantil, não há evidências de que meninos e meninas apresentariam desempenho cognitivo exacerbadamente distintos (Bornstein, Han, \& Haynes, 2004; Nisbett et al., 2012).

Em relação ao conjunto Relacionamento Interpessoal, há indicadores em dois trabalhos que apontariam para mesma direção: no presente estudo há presença mais acentuada de $(\mathrm{H}), \mathrm{Hd}, \mathrm{Hh}$ e PHR, indicando menores recursos de percepção do outro nos meninos, ou então uma percepção mais fantasiosa. No estudo de Carvalho (2015), o mesmo foi observado, ao ver-se aumentado o indicador $\mathrm{Fd}$, apontando para uma percepção mais dependente, menos madura do relacionamento interpessoal. Também no estudo de 
Fernandes (2010) há indicador de que as meninas perceberiam a relação interpessoal de maneira mais ajustada e madura quando comparadas aos meninos (presença de H). Porém, ressalta-se que, no presente estudo, essas variáveis não foram consideradas significativas, dada a ferramenta de correção utilizada. Aplicando-se novamente o método de correção de Bonferroni nos dados de Carvalho (2015) e Fernandes (2010), as variáveis também não seriam consideradas por ultrapassar os valores estimados.

Ao passo que a correção de Bonferroni diminui erro do tipo I, ela poderia aumentar erro do tipo II, ou seja, quando se rejeita a hipótese nula quando ela é falsa. Isso se deve ao rigor e conservadorismo nesse tipo de ferramenta (Abdi, 2007; Armstrong, 2014). Dessa forma, compreende-se que três pesquisas diferentes apontaram para discrepâncias entre meninos e meninas na percepção da relação interpessoal, embora nenhuma seja significativa ao se considerar o ajustamento. Supõe-se que esse seria um caso em que a hipótese nula é falsa, embora ainda sejam necessários mais estudos com a faixa etária para se fortalecer essa hipótese.

No que tange a faixa etária dos 8 aos 9 anos, é possível verificar que, mesmo com o método de correção de Bonferroni, três variáveis permaneceram com índice de significância dentro do estabelecido, $\mathrm{Bl}$ (sangue), XA\% e WDA\%. A primeira delas, Bl que pertence ao grupo Processamento Emocional/Afetivo, é codificada quando o sujeito se refere a sangue como conteúdo da resposta. Essa percepção estaria ligada a conteúdos considerados críticos, ou seja, envolvendo agressividade, podendo refletir a forma como o sujeito se percebe ou como percebe ao outro (Carvalho, 2015; Villemor-Amaral \& Primi, 2009). Os meninos, nessa amostra, produziram mais $\mathrm{Bl}$, podendo estar relacionado a identificação com agressividade, ou maior exposição a contextos que envolvem exposição de conteúdos agressivos.

Em Carvalho (2015), os meninos também apresentaram mais frequentemente conteúdo $\mathrm{Bl}$ (sangue) do que as meninas, mesmo quando controlado o nível de significância pela correção de Bonferroni. Outros dados de Carvalho (2015) apresentam indicadores que vão ao encontro da hipótese de que meninos apresentariam mais frequentemente indicadores de estimulação emocional, ou até dificuldade emocional, tangenciando a agressividade. Mesmo aplicando-se o ajuste de Bonferroni, verifica-se variáveis como C (cor pura) e FM (movimento animal), ambas significando impulsividade e tendência ao descontrole emocional. $\mathrm{O}$ mesmo se observou no estudo de Tackett, Kushner, Herzhoff, Smack e Reardon (2016) em que os autores verificaram maiores pontuações em uma escala que media agressividade em meninos e meninas, observando que estes apresentavam maiores indicadores nesse fator. No estudo de Soto (2015), conscienciosidade (em instrumento de autorrelato baseado no modelo Big Five) nos meninos apareceu baixa, o que pode ter relação indireta com a dificuldade em lidar com estimulação emocional.

Já as duas outras variáveis, $\mathrm{XA} \%$ e WDA\%, foram mais presentes nas meninas e estão relacionadas ao conjunto Mediação. A presença desses indicadores estaria associada à capacidade de processar os estímulos de forma objetiva e coerente com a realidade. Resultados similares foram parcialmente encontrados no estudo de Carvalho (2015) em que as meninas produziram mais $\mathrm{F}$, o que indicaria pensamento mais objetivo e direto. Porém, ao aplicar-se correção de Bonferroni, a variável $\mathrm{F}$ deixaria de ser analisada no estudo da autora. No estudo de Soto (2015), meninas apresentaram menores níveis de abertura à experiência entre os 8 e 9 anos. Esse dado vai em direção ao aqui encontrado, uma vez que XA\% e WDA $\%$ se referem a pensamento mais objetivo e simplificado, sem grandes desenvolvimentos ou diferenciações.

$\mathrm{Na}$ faixa etária dos 10 aos 11 anos, os meninos apresentaram mais respostas do tipo ma (movimento inanimado ativo) e $\mathrm{m}$ (movimento inanimado) quando comparados às meninas. Ambas variáveis são pertencentes ao grupo Processamento Emocional/Afetivo e interpretadas mais especificamente a forma como o sujeito é afetado pelos impulsos e quais seus recursos para controlá-los. O aumento de movimento inanimado está relacionado à presença de atividade mental não deliberada provocada por sentimento de impotência, desesperança e falta de controle sobre as interferências desagradáveis que eventos e pessoas têm sobre si (Carvalho, 2015; Villemor-Amaral \& Primi, 2009; Villemor-Amaral \& Quirino, 2013).

Embora não tenham sido consideradas significativas ao nível de correção de Bonferroni, houve diferença na produção de respostas Ad (detalhe animal), sendo que meninos apresentaram mais respostas desse tipo. $\mathrm{O}$ mesmo foi encontrado por Raspantini (2010) em uma amostra de crianças de nove a 11 anos. Esse indicador estaria relacionado à percepção parcial das relações interpessoais, possivelmente em decorrência de desconfortos emocionais. Esse resultado poderia sugerir o aumento de emoções negativas evidenciado nas variáveis m e ma. Por outro lado, o estudo de Silva e Campos 
(2000) encontrou resultados diferentes, aplicando-se o Rorschach em uma amostra de crianças portuguesas de 10 anos. Os autores verificaram que meninas apresentavam mais frequentemente variáveis relacionadas ao estresse e dificuldades para lidar com estimulação afetiva (aumento de CSBelnd e SumSh). Faz-se a ressalva de que, embora ambos instrumentos sejam similares, há divergências em alguns pontos.

Os resultados de estudos sobre a diferença entre meninos e meninas na percepção do estresse e os recursos para lidar com ele são pouco coesos, sendo que algumas pesquisas verificaram que meninas apresentariam mais recursos, inclusive sociais para lidar com sobrecarga emocional (Van den Akker, Dekovic, Asscher, \& Prinzie, 2014) e outros encontraram resultados opostos (Soto et al., 2011). No estudo de Soto (2015), não foram encontradas diferenças entre meninos e meninas no que tange o afeto das emoções, descontrole emocional e neuroticismo. Essa diferença se iniciaria apenas por volta dos 15 anos.

Por fim, na faixa etária dos 12 aos 14 anos, apenas uma variável apresentou diferença significativa, $\mathrm{Xu} \%$ em maior ocorrência nas meninas. Essa variável, agrupada na área Mediação diz sobre um modo mais subjetivo e idiossincrático de compreender os fatos, mas não necessariamente patológico. $\mathrm{O}$ mesmo se observou no estudo de Carvalho (2015) ao verificar que as meninas na faixa etária dos 13 aos 14 anos produziram mais FQu quando comparadas aos meninos. Essas duas variáveis tem a mesma interpretação uma vez que ambas se baseiam na presença de qualidade formal tipo u. Quando se aplica ajuste de Bonferroni na amostra de Carvalho (2015), a diferença de FQu para meninos e meninas deixa de ser significativamente relevante.

Ao analisar de forma integrada todos os resultados, verifica-se que alguns corroboram com o que já foi encontrado na literatura, tanto em relação a estudos com testes de manchas de tinta quanto com inventários de personalidade. Outros dados, por sua vez, seguiram direções opostas ao previsto em outros estudos. Meyer et al. (2014) argumentam que as diferenças entre meninos e meninas, homens e mulheres seriam decorrentes de outros fatores que não necessariamente o sexo biológico por si só, como é o caso de fatores culturais associados a papéis sociais estereotipados e ensinados a meninos e meninas. Dessa forma, observa-se que, embora haja algumas diferenças no desempenho de meninos e meninas, essas tendem a ser pequenas, muito próximo ao observado em outros testes de manchas de tintas, com a população adulta.
Os dados devem ser considerados com cautela, pois podem estar ocultando relações entre variáveis que não foram investigadas no presente estudo, tais como características sociodemográficas e nível cognitivo. Destaca-se algumas limitações, como o não controle de variáveis sociodemográficas que poderiam interferir nos achados. Além disso, não foi avaliado o nível cognitivo dos participantes e esse fator também poderia impactar na produção de alguns códigos (tríade cognitiva, como feito por Cardoso \& Oliveira, 2018).

Dessa forma, ao analisar estudos com manchas de tinta com outras amostras de crianças, algumas variáveis se repetem, outras, porém, não são verificadas ou até mesmo encontram-se em direção interpretativa oposta. Esse tipo de discrepâncias parece não ser incomum em estudos que buscam verificar diferenças entre meninos e meninas no que tange aos traços de personalidade. $\mathrm{O}$ investimento em novos estudos com maior diversidade de faixas etárias seria um caminho possível para verificar tais discordâncias. Adicionalmente, poderiam ser desenvolvidos estudos longitudinais a fim de verificar o desenvolvimento do funcionamento psicológico de sujeitos ao longo da vida.

\section{Referências}

Abdi, H. (2007). The Bonferroni and Sidák corrections for multiple comparisons. Em N. Salkind, Encyclopedia of measurement and statistics. Sage Publications.

American Educational Research Association, American Psychological Association, \& National Council on Measurement in Education. (2014). Standards for educational and psychological testing. Washington: American Educational Research Association.

Angelini, S. N., \& Oliveira, R. V. (2003). Aplicação do teste verbal Zulliger (forma individual) em pessoas surdas. Psic: Revista de Psicologia da Vetor Editora, 4(1), 82-93.

Armstrong, R. A. (2014). When to use the Bonferroni correction. Ophthalmic \& Physiological Optics, 1-7. doi: 10.1111/opo.12131

Biasi, F. C., \& Villemor-Amaral, A. E. (2016). Evidências de validade do Zulliger-SC para avaliação do relacionamento interpessoal de crianças. Psico (Porto Alegre), 47(1), 13-23. doi: 10.15448/1980-8623.2016.1.19990

Bornstein, M. H., Han, C. H., \& Haynes, O. M. (2004). Specific and general language performance across 
early childhood: Stability and gender considerations. First Language, 24, 267-304.

Branje, S. J. T., Van Lieshout, C. F. M., \& Gerris, J. R. M. (2007). Big Five personality development in adolescence and adulthood. European Journal of Personality, 21, 45-62.

Cardoso, L. M., Gomes, G. V. A., Pacheco, F. P., \& Dias-Viana, J. L. (2018). Análise da produção de artigos científicos brasileiros sobre o Teste de Zulliger. Interação em Psicologia, 22(3).

Cardoso, L. M., Gomes, G. V. A., \& Vieira, T. S. (2018). Evidências de Validade do Zulliger-SC para Avaliação de Crianças. Psico-USF, 23(3), 451-460. doi: 10.1590/1413-82712018230305

Cardoso, L. M., \& Oliveira, J. C. (2018). Meninos e meninas: Influências culturais no método de Zulliger. Avaliação Psicológica, 17(1). doi: 10.15689/ ap.2017.1701.11.13361

Carvalho, A. C. M. (2015). Normatização do teste de Zulliger SC para crianças e adolescentes (Dissertação). Pontifícia Universidade Catolica de Goiás, Goiás.

Carvalho, A. C. M., \& Resende, A. C. (2018). Desempenho de Crianças e Adolescentes Não Pacientes no Zulliger SC. Avaliação Psicológica, 17(1), 142-154. doi: 10.15689/ap.2017.1701.15.13795

Caspi, A., Roberts, B. W., \& Shiner, R. L. (2005). Personality Development: Stability and Change. Annual Review of Psychology, 56, 453-484. doi: 10.1146/annurev.psych.55.090902.141913

Chabert, C. (2004). Psicanálise e métodos projetivos. São Paulo: Vetor.

Cohen, J. (1992). A power primer. Psychological Bulletin, 112(1), 155-159. doi: 10.1037/0033-2909.112.1.155

Cury-Jacquemin, R. C. de P. (2012). Padrões normativos do Psicodiagnóstico de Rorschach em adolescentes de 12 a 14 anos (Dissertação). Universidade de São Paulo, Ribeirão Preto.

Denham, S. A., Wyatt, T. M., Bassett, H. H., Echeverria, D., \& Knox, S. S. (2009). Assessing social-emotional development in children from a longitudinal perspective. Journal of Epidemiology \& Community Health, 63(Suppl 1), i37-i52. doi: 0.1136/jech.2007.070797

Di Domenico Grazziotin, J. B. D., \& Scortegagna, S. A. (2016). Revisão de pesquisas brasileiras sobre o
Teste de Zulliger publicadas em artigos. Avaliação Psicológica, 15(2). doi: 10.15689/ap.2016.1502.11

Fernandes, S. (2010). Normas do Rorschach em crianças de seis a oito anos (Dissertação). Universidade de São Paulo, Ribeirão Preto.

Ferreira, M. E. A., \& Villemor-Amaral, A. E. (2005). $O$ teste de Zulliger e avaliação de desempenho. Paidéia, 15(32), 367-376. doi: 10.1590/ S0103-863X2005000300006

Franco, R. R. C., \& Villemor-Amaral, A. E. (2012). Validade incremental do Zulliger e do Pfister no contexto da toxicomania. Psico-USF, 17(1), 73-83. doi: 10.1590/S1413-82712012000100009

Gregoleti, V., \& Scortegagna, S. A. (2017). The ZulligerCS in elderly on hemodialysis and the relationship between external variables. Paidéia, 27(66), 43-50. doi: 0.1590/1982-43272766201706

Heaven, P. C. L. (1996). Personality and Self-Reported Delinquency: A Longitudinal Analysis. The Journal of ChildPsychology andPsychiatry. doi: 10.1111/j.14697610.1996.tb01467.x

Klimstra, T. A., Hale III, W. W., Raaijmakers, Q. A. W., Branje, S. J. T., \& Meeus, W. H. J. (2009). Maturation of personality in adolescence. Journal of Personality and Social Psychology, 96(4), 898-912. doi: $10.1037 /$ a0014746

Meyer, G. J., Giromini, L., Viglione, D. J., Reese, J. B., \& Mihura, J. L. (2014). The association of gender, ethnicity, age, and education with Rorschach scores. Assessment, 22, 1-19. doi: $0.1177 / 1073191114544358$

Nascimento, R., Pedroso, J. S., \& Souza, A. M. (2009). Método de Rorschach na avaliação psicológica de crianças: Uma revisão de literatura. Psico-USF, 14(2), 193-200. doi: 10.1590/S1413-82712009000200008

Nisbett, R. E., Aronson, J., Blair, C., Dickens, W., Flynn, J., Halpern, D. F., \& Turkheimes, E. (2012). Intelligence: New findings and theoretical developments. American Psychologist, 67(2), 130-159. doi: 10.1037/ a0026699

Raspantini, R. L. (2010). O psicodiagnóstico de Rorschach em crianças de 9 a 11 anos: Um estudo normativo (Dissertação). Universidade de São Paulo, Ribeirão Preto.

Resende, A. C., Carvalho, T. C. R., \& Martins, W. (2012). Desempenho médio de crianças e adolescentes 
no método de Rorschach Sistema Compreensivo. Avaliação Psicológica, 11(3), 375-394.

Ribeiro, R. K. S. M., Semer, N. L., \& Yazigi, L. (2011). Rorschach Comprehensive System norms in brazilian children from public and private schools. Psicologia: Reflexão e Crítica, 24(4), 671-684.

Rodrigues, E. C., \& Alchieri, J. C. (2009). Avaliação das características de afetividade em crianças e jovens com síndrome de Down. Psico-USF, 14(1), 107116. doi: 10.1590/S1413-82712009000100011

Ruiz, M. A., Pincus, A. L., \& Dicknson, K. A. (2003). NEO PI-R Predictors of Alcohol Use and Alcohol-Related Problems. Journal of Personality Assessment, 81(3), 226-236. doi: 10.1207/ S15327752JPA8103_05

Silva, D. R., \& Campos, R. (2000). Some Rorschach variables from a normative study with 10 -year-old Portugues children. Rorschachiana, 24, 110-126. doi: 10.1027/1192-5604.24.1.110

Soto, C. J. (2015). The little six personality dimensions from early childhood to early adulthood: Mean-level age, and gender differences in parents' reports. Journal of Personality, 1-14. doi:10.1111/jopy.12168

Soto, C. J., John, O. P., Gosling, S. D., \& Potter, J. (2011). Age differences in personality traits from 10 to 65: Big five domains and facets in a large crosssectional sample. Journal of Personality and Social Psychology, 100(2), 330-348. doi: 10.1037/a0021717

Tackett, J., Kushner, S. C., Herzhoff, K., Smack, A. J., \& Reardon, K. (2016). Viewing relational aggression through multiple lenses: Temperament, personality, and personality pathology. Development and Psychopathology, 26, 863-877. doi: 10.1017/ S0954579414000443

Tavella, R. R., \& Villemor-Amaral, A. E. (2014). O teste de Zulliger-SC: Avaliação da criatividade em crianças. Estudos de Psicologia (Campinas), 31(4), 489-497. doi: 10.1590/0103-166X2014000400003
Van den Akker, A. L., Dekovic, M., Asscher, J., \& Prinzie, P. (2014). A temporary defiance of the maturity principle and bidirectional associations with parenting. Journal of Personality and Social Psychology, 107(4), 736-750. doi: 10.1037/a0037248

Vaz, C. E. (2000). A técnica de Zulliger no processo de avaliação da personalidade. In J. A. Cunha (Org.), Psicodiagnóstico-V (5 ed, p. 386-387). Porto Alegre: Artmed.

Vaz, C. E. (2004). Z-teste técnica de Zulliger forma coletiva sistema Klopfer: Pesquisas básicas no Brasil. São Paulo: Casa do Psicólogo.

Villemor-Amaral, A. E., \& Machado, M. A. S. (2011). Indicadores de depressão do Zulliger no Sistema Compreensivo (ZSC). Paidéia, 21(48), 21-27. doi: 0.1590/S0103-863X2011000100004

Villemor-Amaral, A. E., Pavan, P. M. P., Tavella, R. R., Cardoso, L. M., \& Biasi, F. C. (2016). Validity evidence of the Z-Test-SC for use with children. Paidéia (Ribeirão Preto), 26(64), 199-206. doi: 10.1590/1982-43272664201607

Villemor-Amaral, A. E., \& Primi, R. (2009). Teste de Zulliger no sistema compreensivo, ZSC: Forma individual. São Paulo: Casa do Psicólogo.

Villemor-Amaral, A. E., \& Quirino, G. de S. (2013). Estudo comparativo entre indicadores afetivos das técnicas de Pfister e Zulliger. Avaliação Psicológica, 12(1), 1-7.

Villemor-Amaral, A. E., Santos, M. A., \& Noronha, A. P. P. (2009). O Zulliger no Sistema Compreensivo: Um estudo de fidedignidade. Psicologia: Ciência e Profissão, 29(4), 656-671.

Villemor-Amaral, A. E., \& Vieira, P. G. (2016). Zulliger (CS) in assessing the relational maturity of children. 26(65), 369-376. doi: 0.1590/1982-43272665201601

Recebido em: 24/04/2019

Reformulado em: 20/03/2020

Aprovado em: 17/04/2020 
Sobre os autores:

Ana Carolina Zuanazzi é Graduada em Psicologia pela Universidade Estadual de Londrina (2014), especialista em Neuropsicologia pelo Centro de Diagnóstico Neuropsicológico (2015) e Mestre em Psicologia Clínica pela Universidade de São Paulo (2015) e Doutora em Psicologia com ênfase em avaliação psicológica pela Universidade São Francisco (2019), com estágio na University of Toledo-Ohio. Atualmente é Gerente de Projetos na área de Geração de Evidências no Instituto Ayrton Senna. Atua principalmente nos seguintes temas: Avaliação Psicológica, Técnicas Projetivas, Rorschach (R-PAS), Inteligência Emocional e competências Socioemocionais. É proficiente em Administração e Codificação pelo Rorschach Performance Assessment System (R-PAS).

ORCID: https://orcid.org/0000-0003-1649-2372

E-mail: anacarolina.zf@gmail.com

André Pereira Gonçalves possui graduação em Psicologia pela Universidade José do Rosário Vellano (2015). Especialista em Avaliação Psicológica pela PUC campus Poços de Caldas-MG (2017). Mestre em Psicologia com área de concentração em Avaliação Psicológica pela Universidade de São Francisco com bolsa CAPES (2018). Doutorando do programa stricto sensu de pós graduação em Psicologia da Universidade São Francisco com bolsa CAPES e Membro do Laboratório de Avaliação Psicológica em Saúde Mental (LAPSaM).

ORCID: https://orcid.org/0000-0002-2470-4040

E-mail: andregoncalvespsi@gmail.com

Anna Elisa Villemor Amaral é Graduada em Psicologia pela PUC-SP. Mestrado e Doutorado em Ciências pela Universidade Federal de São Paulo (UNIFESP/EPM) e pós-doutorado na Universidade da Savoia/ França. Professora Associada Doutora do Programa de Pós-Graduação Strico Sensu em Psicologia da Universidade São Francisco e líder do grupo de pesquisa Avaliação Psicológica em Saúde Mental no CNPq. É Bolsista Produtividade CNPq 1C. Foi membro da Comissão Consultiva em Avaliação Psicológica do SATEPSI/ CFP (2010 a 2012). Presidiu a Associação Brasileira de Rorschach e Métodos Projetivos (2002 a 2006), é hoje é membro de seu conselho consultivo. Foi coordenadora do GT de Métodos Projetivos da ANPEPP( 2008 a 2012). Assessora ad hoc CNPq, FAPESP e CAPES. É coordenadora do Laboratório de Avaliação Psicológica em Saúde Mental - LAPSaM. Editora Associada da revista Rorschachiana. Membro do Comitê de Ética em Pesquisa da Universidade São Francisco (CONEP). Membro do Comitê Assessor de Psicologia no CNPq.

ORCID: https://orcid.org/0000-0002-1815-8194

E-mail: aevillemor@gmail.com 
Fabiano Koich Miguel possui graduação em Psicologia pela Universidade Presbiteriana Mackenzie (2002) e especialização em Psicologia do Trânsito pela Universidade Cruzeiro do Sul (2003). Concluiu mestrado (2006) e doutorado (2010) em Avaliação Psicológica pela Universidade São Francisco, com doutorado-sanduíche na Universidade de Évora (Portugal) e na University of Toledo (EUA), desde então colaborando no Rorschach Performance Assessment System (R-PAS). Tem experiência em clínica e na área acadêmica, atuando principalmente com os seguintes temas: construção de instrumentos, inteligência emocional e personalidade, testagem adaptativa informatizada. Atualmente é professor associado da Universidade Estadual de Londrina (UEL), sendo docente na graduação e no mestrado em Psicologia. É parecerista ad hoc da Comissão Consultiva em Avaliação Psicológica do Conselho Federal de Psicologia, sendo também membro da Comissão em 2013. Foi pesquisador convidado na Università degli Studi di Torino (Itália) em 2015-2016 e na University of Toledo (EUA) em 2018. Participa do Grupo de Trabalho da ANPEPP (GT) Avaliação Psicológica: Personalidade e Desenvolvimento Humano.

ORCID: https://orcid.org/0000-0003-2498-692X

E-mail:fabiano@avalpsi.com.br

Contato com os autores:

anacarolina.zf@gmail.com 